\title{
PREPARATION OF RARE EARTH - TRANSITION METAL (RE: Y, TM: CO) INTERMETALLIC COMPOUNDS BY CALCIOTHERMIC REDUCTION DIFFUSION PROCESS
}

\author{
M. Ilayaraja ${ }^{1}$, L. John Berchmans ${ }^{2}$, Sankara Raman Sankaranarayanan ${ }^{1 *}$ \\ ${ }^{1}$ Department of Metallurgical and Materials Engineering, \\ National Institute of Technology Tiruchirappalli, \\ Tiruchirappalli 620 015, Tamil Nadu, INDIA \\ ${ }^{2}$ Principal Scientist, Electro - Pyro Metallurgy Division, \\ CSIR-CECRI, Karaikudi, Tamil Nadu, INDIA
}

Received 17.11.2013

Accepted 16.12.2013

\begin{abstract}
Rare earth cobalt alloys have many special magnetic properties and can be used to prepare magnetic and magneto-optical components. The yttrium - cobalt intermetallic compounds are prepared by calciothermic reduction - diffusion (CRD) process at temperature of $1000^{\circ} \mathrm{C}$, under argon atmosphere. Yttrium oxide, metallic cobalt powder, metallic calcium are used as raw materials in this process. Calcium acts as the reductant, which is used to prepare the YCo5 magnetic material. XRD, SEM, EDAX and some thermodynamic valuation have been carried out on the products. The chemical reactions controlled by unreacted core model theory were studied.

Keywords: Calciothermic reduction process (CRD), Intermetallics, Rare earth Cobalt alloys, Gibbs free energy
\end{abstract}

\section{Introduction}

Permanent magnetic materials have revolutionized technology; and are of growing interest to the electronics industry and to manufacturers of electro-mechanical devices, with applications in many electronic apparatus, circuits, electric motors, consumer, and defence products. These magnets have major influence on the size, efficiency, stability, and cost of cited devices. The rare earth alloy magnets that have been most extensively studied and developed are $\mathrm{SmCo}$ and $\mathrm{NdFeB}$. There are three families of rare earth magnets commercially available, namely $\mathrm{SmCo}_{5}, \mathrm{Sm}_{2} \mathrm{Co}_{17}$ and $\mathrm{Nd}_{2} \mathrm{Fe}_{14} \mathrm{~B}$ based magnets, which were developed in the 1960s, 1970s and 1980s, respectively [1]. In recent years, a new generation of lanthanide-based intermetallic materials have emerged, with excellent magnetic properties and also primary interest is

*Corresponding Author: Sankara Raman Sankaranarayanan , raman@nitt.edu 
focused on the light lanthanides, i.e. $\mathrm{La}, \mathrm{Ce}, \mathrm{Pr}, \mathrm{Nd}, \mathrm{Sm}$ and $\mathrm{Y}$, even though the light lanthanides generally have smaller magnetic moments than the heavy lanthanides. In the $\mathrm{LnCo}_{5}$ structure, the heavy lanthanide moment couples antiferromagnetically with the cobalt moment, reducing the saturation magnetization.

There are four major possible routes for the production of rare earth-cobalt alloys. These are (1) arc melting, (2) induction melting, (3) electro-winning, and (4) reduction-diffusion process. Amongst these, the first two methods are well established and widely practiced. These methods are simple to operate and yield alloys of the highest purity. However, both these processes require the alloy constituents in their elemental form/s, and hence turn out to be quite expensive [2]. The reduction-diffusion (RD) process is a method by which the rare earth-transition metal intermetallic compound powders can be prepared over a relatively wide temperature range using the cheap rare earth oxides, transition metal powder and calcium (or $\mathrm{CaH}_{2}$ ) as the raw materials. In many cases, calcium hydride $\left(\mathrm{CaH}_{2}\right)$ has been used as reducing agent [2].

The advantages of the reduction-diffusion (RD) method are the direct use of rare earth oxides as raw materials, low cost, and elimination of the homogenization process for the elimination $\alpha-\mathrm{Fe}$ phase in the ingot. The RD process is relatively less capital intensive, easy to operate and straightaway yields alloys of the desired composition [3]. Therefore, the RD process is a promising technique, with low cost for the preparation of magnetic materials. In the present study, the proposed Y-Co alloy has been produced using the calciothermic reduction-diffusion process.

\section{Experimental Procedure}

The raw materials used in experiments are powders of yttrium oxide $\left(\mathrm{Y}_{2} \mathrm{O}_{3}\right)$, metallic cobalt powder and calcium granules with purity of $99.55 \%$ in mass content as stoichiometric ratio. These powders were mixed and pressed into pellets, at pressure of 1.44 tons $/ \mathrm{cm}^{2}$. The pellet was then placed in a high density alumina crucible, which was placed in high temperature electrical furnace at $1000{ }^{\circ} \mathrm{C}$ for 7 hours, under argon atmosphere. After the reaction, the product was cooled to room temperature and the sample was taken from the crucible and then washed with 5\% dilute acetic acid and distilled water subsequently, to remove the impurities and $\mathrm{CaO}$. The desired $\mathrm{YCo}_{5}$ powder was weighed to calculate the rate of reduction - diffusion, also called as a "conversion rate" $(\alpha)[4]$.

Rate of reduction-diffusion may be expressed as:

$(\alpha)=\mathrm{M}_{\mathrm{Y}} / \mathrm{M}_{\mathrm{Y}}^{\circ} \mathrm{x} 100 \%$

where $\mathrm{M}_{\mathrm{Y}}$ is the weight of $\mathrm{Y}$ in $\mathrm{YCo}_{5}$ formed by the diffusion of $\mathrm{Y}$ into $\mathrm{Co}$, and $\mathrm{M}^{\circ}{ }_{\mathrm{Y}}$ is the weight of $\mathrm{Y}$ in the raw materials [3].

\section{Mechanism of reaction}

Mechanism of the reaction is defined by the reaction:

$\mathrm{Y}_{2} \mathrm{O}_{3}+10 \mathrm{Co}$ (powder) $+3 \mathrm{Ca}$ (granules) $\rightarrow 2 \mathrm{YCo}_{5}+3 \mathrm{CaO}$

From the above equation, it is understood that $\mathrm{Y}_{2} \mathrm{O}_{3}$ can be reduced by $\mathrm{Ca}$, and then $\mathrm{Y}$ diffuses in the matrix of $\mathrm{Co}$ forming $\mathrm{YCo}_{5}$. The process consists of two steps:

(1) Under the elevated temperature, Ca vapour quickly diffuses to the surface of $\mathrm{Y}_{2} \mathrm{O}_{3}$, where $\mathrm{Y}_{2} \mathrm{O}_{3}$ is reduced by $\mathrm{Ca}$; and $\mathrm{Y}$ is produced. 
(2) The Y produced diffuses to the adjacent surface of Co powder to form $\mathrm{YCo}_{5}$.

The reaction continues from the exterior to the interior, so it can be controlled by unreacted core model theory. The formula is derived from the relationship between conversion rate and time.

If the reaction is controlled by gas phase transferring reaction [3], then:

$\mathrm{kt}=\alpha$ [3], then:

In the case if the reaction is controlled by gas-solid interface chemical reaction

$\mathrm{kt}=1-(1-\alpha)^{1 / 3}$

If the reaction is controlled by resultant layer internal diffusion [3], then the following equation may be applied:

$\mathrm{kt}=1-3(1-\alpha)^{2 / 3}+2(1-\alpha)$

where, $\alpha$ - conversion rate, $\mathrm{t}$ - reaction time, $\mathrm{k}$ - reaction rate constant. Using Eq. (5), we obtained the rate constant for internal diffusion of this system as $8.25 \times 10^{-7} \mathrm{sec}^{-1}$.

\section{Thermodynamic Aspects}

In the $\mathrm{RD}$ process for $\mathrm{YCo}_{5}$ preparation, the following reaction was considered:

$\mathrm{Y}_{2} \mathrm{O}_{3(\mathrm{~s})}+10 \mathrm{Co}_{(\mathrm{s})}+3 \mathrm{Ca}_{(\mathrm{g})} \rightarrow 2 \mathrm{YCo}_{5(\mathrm{~S})}+3 \mathrm{CaO}_{(\mathrm{s})}$

Assuming, the $\mathrm{YCo}_{5}$ is an ideal solution, the change in Gibbs free energy due to formation of a solution of mixing [5] is given by:

$\Delta \mathrm{G}^{\mathrm{M}}{ }_{298}=\mathrm{RT}\left(\mathrm{XA} \ln \mathrm{a}_{\mathrm{A}}+\mathrm{X}_{\mathrm{B}} \ln \mathrm{a}_{\mathrm{B}}\right)$

For an ideal solution, $\mathrm{a}_{\mathrm{A}=\mathrm{Xa}}$

$\mathrm{Y}+5 \mathrm{Co}=\mathrm{Y} \mathrm{Co}_{5}$

$\mathrm{Y}_{2} \mathrm{O}_{3}+3 \mathrm{Ca}=2 \mathrm{Y}+3 \mathrm{CaO}$

$\mathrm{Ca}_{(\mathrm{l})}=\mathrm{Ca}_{(\mathrm{g})}$

Rearranging the equations (8), (9) and (10), the Gibbs free energy of mixing of solution at $1273 \mathrm{~K}$ was calculated. The change in Gibbs free energy due to formation of a solution at $1273 \mathrm{~K}$ is found to be $-568.971 \mathrm{KJ} /$ mole.

\section{Characterization}

X-ray powder diffraction (XRD) patterns were recorded with a PW3040/60 X'pert PRPANalytical, Netherlands diffractometer with $\mathrm{Cu} \mathrm{K} \mathrm{K}_{\alpha}$ radiation. The morphology was examined with a scanning electron microscope (SEM) Hitachi. Chemical composition of the samples was examined by energy dispersive-X-ray analyzer (EDAX). 


\section{Results and Discussion}

\section{XRD Analysis}

The XRD patterns were obtained for the specimens produced by reaction at $1273 \mathrm{~K}$ for 7 hours. The well defined peaks of $\mathrm{YCo}_{5}$ and $\mathrm{CaO}$ and other impurities observed in this system are shown in Figure 1. equation:

The average crystalline size of samples was calculated according to the following

$$
\mathrm{T}=\mathrm{k} * \lambda / \mathrm{B} * \cos \theta_{\mathrm{B}}
$$

where, $\mathrm{T}=$ average crystalline size of the samples, $\mathrm{K}=$ constant dependent on crystallite shape (0.89), $\Lambda=$ X-ray wave length $(1.54 \mathrm{~nm}), \mathrm{B}=\mathrm{FWHM}$ (full width at half $\max )$, and $\theta_{\mathrm{B}=}$ Bragg angle respectively.

The full-width at half-maximum (FWHM) of the $\mathrm{YCo}_{5}$ peak was used in the Scherrer equation (11) to independently estimate the average crystalline size of $\mathrm{YCo}_{5}$, ranging from $0.1 \mu \mathrm{m}$ to $0.6 \mu \mathrm{m}$.

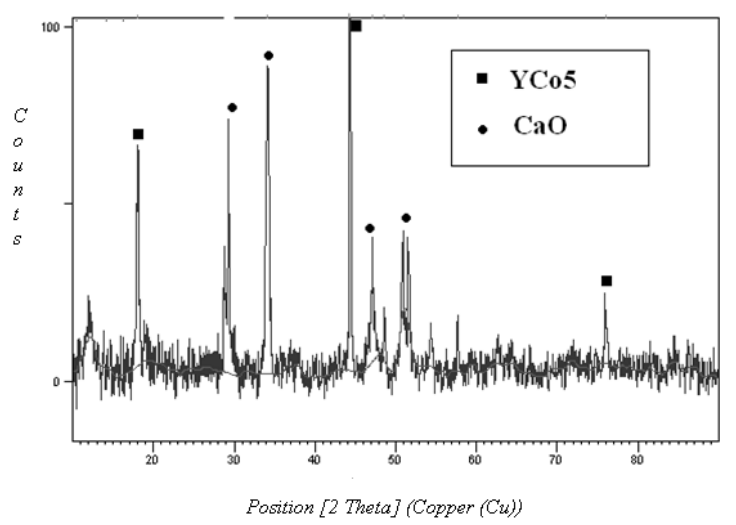

Figure 1. XRD peaks of $\mathrm{YCO}_{5}$ produced at $1000^{\circ} \mathrm{C}$

SEM Analysis

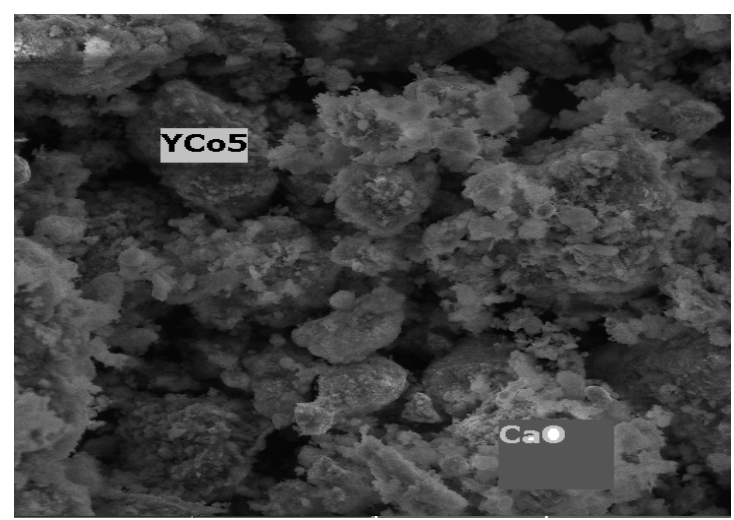

Figure 2. (SEM). Morphology of $\mathrm{YCo}_{5}$ produced at $1000^{\circ} \mathrm{C}$ 
SEM micrographs revealing the particle size and morphology of the prepared crystals are shown in Figure 2. The $\mathrm{YCo}_{5}$ grains in the pellets were examined after the calciothermic reduction-diffusion process. The rare-earth - intermetallic products $\left(\mathrm{YCo}_{5}\right)$ were found to be hexagonal in shape. The morphology of $\mathrm{YCo}_{5}$ and minor impurity $(\mathrm{CaO})$ was examined. The $\mathrm{YCo}_{5}$ particle size analysis has been carried out at $20 \mu \mathrm{m}$ standard size.

\section{EDAX analysis}

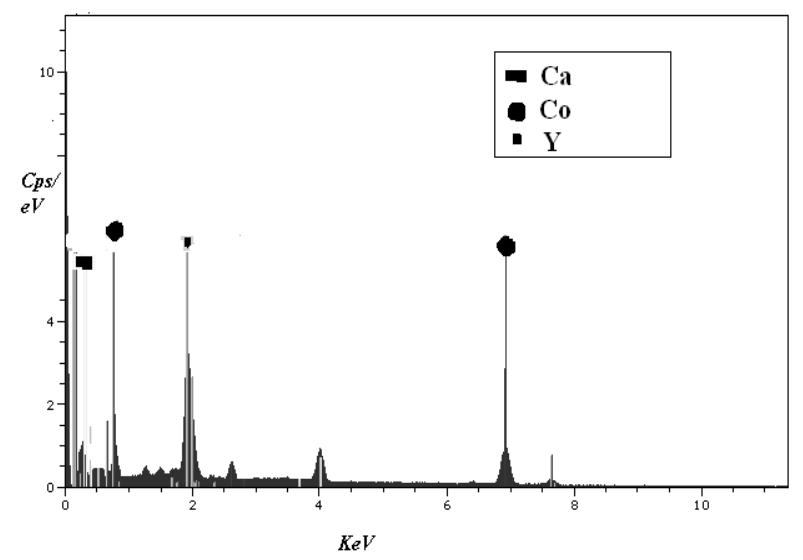

Figure 3. EDAX of $\mathrm{YCo}_{5}$ at produced at $1000^{\circ} \mathrm{C}$

Chemical composition has been observed by EDAX technique after calciothermic reduction-diffusion process and presented in Figure 3. The result analyzed with EDAX shows that the atomic percentage-wise composition of $\mathrm{YCo}_{5}$ was $\mathrm{Y}=15.0$ and $\mathrm{Co}=75 \%$, respectively.

\section{Conclusions}

In $\mathrm{Ca}-\mathrm{Y}_{2} \mathrm{O}_{3}$-Co system, it is possible to produce $\mathrm{YCo}_{5}$ alloy by reductiondiffusion process. The reduction process can be described by contracting core model. The rate constant is $8.25 \times 10^{-7} \mathrm{sec}^{-1}$ (for 7 hours) was required for the conversion of reactants into the products. The Gibbs free energy of mixing of solution at $1273 \mathrm{~K}$ is $568.971 \mathrm{KJ} / \mathrm{mol}$ required to reduce the yttria and diffuses through the Co layer to form a $\mathrm{YCo}_{5}$. The EDAX analysis shows that the atomic percentage of $\mathrm{YCo}_{5}$ were $\mathrm{Y}=15.0$ and $\mathrm{Co}=75 \%$, respectively. The SEM image shows that the morphological and features of the synthesizing crystal and particle size is in the range of $20 \mu \mathrm{m}$. From the XRD results, the presence of pure $\mathrm{YCo}_{5}$ alloy can be ascertained, along with minor impurity like $\mathrm{CaO}$ in the system. The reaction goes on from exterior to interior, so it can be observed by unreacted core model theory.

\section{Acknowledgment}

The authors would like to thank the management of National Institute of Technology Tiruchirappalli and the Director of CSIR-CECRI for their help towards accomplishing this research work. We sincerely thank MHRD, Government of India, for 
providing stipend to the research scholar (Mr Ilayaraja), to carry out the research work. We also acknowledge the MoU between NIT Tiruchirappalli and CSIR - CECRI, which enabled joint research.

\section{References}

[1]. S.R. Trout, Permanent Magnets based on the Lanthanides, www. Spontaneousmaterials.com

[2]. T.S. Krishnan, Bulletin of Materials Science, Vol 2, Number 3, August 1980, pp.161-165.

[3]. Guangsi Guo, Guangqiang Li, and Liang Liu, J. Mater. Sci. Technol., Vol. 16, No. 2, 2000, 181-182.

[4] Guangsi Guo, Guangtai Wang, and Zhitong Sui, J. Mater. Sci. Technol., Vol. 20, No. 1, 2004, 68-70.

[5]. A. Ghosh, Text of Materials and Metallurgical Thermodynamics, $3^{\text {rd }}$ printing, PHI Learning Private Limited, 2009. 